\title{
Realismo Estructural Óntico y estructuras físicas
}

\author{
Bruno Borge \\ Universidad de Buenos Aires \\ Facultad de Filosofia y Letras \\ Puan 480 CABA CABA 1406 \\ Argentina \\ brunojborge@gmail.com \\ borge@filo.uba.ar
}

\section{Article info}

CDD: 501

Received: 25.03.2017; Accepted: 12.06.2017

DOI: http://dx.doi.org/10.1590/0100-6045.2017.V40N2.BB

\author{
Keywords / Palavras clave \\ Ontic Structural Realism / Realismo Estructural Óntico \\ Physical Structure / Estructura física \\ Physicalism / Fisicalismo \\ Scientific Structuralism / Estructuralismo científico
}

\begin{abstract}
Ontic Structural Realism (OSR) states that the ontology of the physical world must be (re)conceived in order to take pure structures are its basic entities. Objects and properties must be eliminated in favor of structures. However, its proponents claim that these pure structures are suitable to account of physical reality, either because they identify the physical world with a mathematical structure, or because they maintain that the structures posited by OSR are not abstract structures, but physical structures. In this paper, I critically analyze both strategies in order to show their limitations. In addition, I propose a new physcalist framework suitable for structuralist ontologies.
\end{abstract}

\section{RESUMEN}

El Realismo Estructural Óntico (REO) afirma que la ontología del mundo físico debe ser (re)concebida de modo que las puras estructuras sean sus entidades básicas. Objetos y propiedades deben ser eliminados en favor de las estructuras. No obstante, sus defensores afirman que dichas puras estructuras son capaces de dar cuenta de la realidad física, o bien porque identifican el mundo físico con una estructura matemática, o bien porque sostienen que las estructuras postuladas por el REO no son estructuras abstractas, sino físicas. En el presente trabajo analizo críticamente ambas estrategias a fin de mostrar sus limitaciones. Asimismo, propongo un nuevo marco fisicalista, adecuado para las ontologías estructuralistas.

Manuscrito - Rev. Int. Fil. Campinas, v. 40, n. 2, pp. 71-97, abr.-jun. 2017. 


\section{Introducción}

El Realismo Estructural Óntico (REO), presentado por Ladyman (1998), afirma que la ontología del mundo físico debe ser (re)concebida de modo que las puras estructuras sean sus entidades básicas ${ }^{1}$. Objetos y propiedades deben ser eliminados en favor de las estructuras. No obstante, sus defensores afirman que dichas puras estructuras son capaces de dar cuenta de la realidad física. Las motivaciones para ese cambio de ontología provienen de dos frentes: la particular naturaleza de las entidades fundamentales postuladas por la física moderna, y la posibilidad de solucionar algunos problemas heredados del debate realismo vs. antirealsmo científicos. Dichas motivaciones, y la consecuente necesidad o conveniencia de un cambio de ontología, han sido ampliamente discutidas en la literatura especializada. Del mismo modo, varios críticos cuestionaron diversos aspectos del REO, desde su mera inteligibilidad como posición filosófica hasta su conveniencia metodológica. Estas discusiones no serán abordadas aquí ${ }^{2}$. Una de las objeciones clásicas al REO, sin embargo, tiene especial interés. Cao (2003, p. 89) y van Fraassen (2006a, p. 292-293), entre otros, señalan que el cambio de ontología implica la disolución de lo físico en lo matemático y, consecuentemente, la imposibilidad del REO de dar cuenta de la realidad física.

Las reacciones a esta objeción por parte de los defensores del REO siguieron dos estrategias.

Tegmark (2008) muerde la bala respecto de la primera parte de la objeción, y acepta que el REO colapsa en el puro platonismo matemático. Sin embargo, en su opinión, hay buenos argumentos para sostener que nuestro universo debe identificarse con una estructura matemática. De ese modo, si bien admite que el REO disuelve lo físico en lo matemático, niega

${ }^{1}$ En sentido estricto, sólo las variantes eliminativistas del REO sostienen esta tesis. Hay versiones no-eliminativistas de esta posición -como la defendida por Esfeld (2009)- que afirman que la estructura es ontológicamente prioritaria respecto de los objetos y propiedades, pero no niegan a éstos existencia. Dado que las versiones eliminativistas del REO son las más extendidas y discutidas, este trabajo, este trabajo se centrará sólo en ellas.

${ }^{2}$ Para un panorama general acerca del REO, véase [BORGE] (2013).

Manuscrito - Rev. Int. Fil. Campinas, v. 40, n. 2, pp. 71-97, abr.jun. 2017. 
que eso sea un obstáculo para dar cuenta de la realidad física, puesto que la realidad física es matemática. El resto de los defensores del REO, en cambio, se concentra en negar de plano la primera imputación. Entienden que las estructuras a las que la nueva ontología refiere no son estructuras matemáticas, sino estructuras físicas, cuyo rasgo característico está dado por su carácter modal.

En el presente trabajo analizo críticamente ambas estrategias a fin de mostrar sus limitaciones. Asimismo, rastreo los orígenes de la tesis fisicalista fuera de los debates sobre el realismo científico. Sostengo que ninguna de las posiciones en juego es adecuada a los fines del realista. Propongo un nuevo marco fisicalista, adecuado para las ontologías estructuralistas.

\section{La Hipótesis del Universo Matemático}

La postulación de estructuras relacionales como el componente básico de la realidad ha levantado sospechas respecto de que el REO pueda afirmarse como una posición acerca del mundo físico. Psillos (2006) ha objetado que una ontología de puras relaciones desemboca inevitablemente en el platonismo. Su preocupación concierne a la naturaleza de las relaciones: si las relaciones son abstracciones respecto de las entidades o propiedades físicas que las instancian, al eliminar estas últimas se eliminan también las primeras. La obvia solución es considerar que dichas relaciones, qua universales, no dependen de los relata para existir. El REO conduce así a una ontología de puros universales platónicos.

Por su parte, van Fraassen (2006a, p. 292-293) pone en duda que el REO sea capaz siquiera de sostener una distinción entre lo que es estructura y los aspectos cualitativos del mundo.

Una tercera línea de argumentación (en la que podría ubicarse a Cao, 2003) apunta al tipo de consecuencias ontológicas que los defensores del REO sacan de su propuesta epistémica. Concretamente, el REO hereda del realismo estructural de Worrall (1989) la doctrina de que sólo conocemos la estructura. Esa estructura es caracterizada a partir del conocimiento de la física moderna que, como los defensores del REO gustan admitir, está

Manuscrito - Rev. Int. Fil. Campinas, v. 40, n. 2, pp. 71-97, abr.jun. 2017. 
altamente matematizada. Así la "estructura del mundo" puede ser descrita en términos puramente matemáticos. Pero dado que el REO va más lejos al afirmar que nuestro conocimiento se limita a la estructura porque ella es lo único que hay, pasa a comprometerse con el correlato físico del formalismo matemático que resulta relevante para fijar la ontología de la ciencia. Ese correlato no pueden ser propiedades u objetos, y dado que lo único relevante aquí para comprender los compromisos ontológicos de la física o la matemática es la estructura, parece entonces no haber diferencia entre las nociones de estructura física y estructura matemática. De ese modo, el REO colapsaría en el platonismo matemático.

Tegmark (2008) acepta de buen grado que el REO debe asumir una ontología de puras entidades matemáticas. Sin embargo, niega que eso sea un escollo para que dicha posición se constituya como un realismo acerca del mundo físico, ya que, entiende, la misma realidad física debe identificarse con una estructura matemática.

Su argumento parte de considerar lo que, sostiene, es la doctrina básica de toda forma realismo. La denomina Hipótesis de la Realidad Externa (HRE): existe una realidad física externa e independiente de los humanos y sus prácticas. Asimismo, de acuerdo con el rumbo que la empresa científica ha tomado, nuestro modo de dar cuenta de forma completa de esa realidad implicaría la formulación de una Teoría del Todo (TdT). Seguramente, habría más de un modo de enunciar esa teoría, como hay más de un modo de expresar cualquier teoría. Lo que interesa a Tegmark es la posibilidad de expresar una TdT de modo que quede libre de todo "bagaje" interpretativo humano, es decir, de un modo que resultase comprensible, por ejemplo, para una civilización alienígena:

Nuestra descripción de las entidades que existen en la realidad externa y las relaciones entre ellas tendrían que ser completamente abstractas, cualquier palabra u otro símbolo utilizado para denotar estaría forzado a ser una mera etiqueta que no tenga significado preconcebido (TEGMARK, 2008, p. 104).

La respuesta a la pregunta acerca de cuál es el lenguaje privilegiado en el que debería expresarse una TdT resulta entonces obvia: "una estructura matemática es precisamente eso: entidades abstractas con relaciones entre ellas" (2008, p. 104).

Manuscrito - Rev. Int. Fil. Campinas, v. 40, n. 2, pp. 71-97, abr.jun. 2017. 
Considero que algunas reservas al curso de su argumento podrían aparecer ya desde este punto. En primer lugar, la posibilidad de aplicar una teoría a un sistema físico (en este caso al sistema físico total que es nuestro universo) parece descansar sobre la consideración de ciertas condiciones iniciales y la posibilidad de registrar de algún modo los resultados observacionales de la experimentación. Es al menos dudoso que ambas cosas puedan ser "absorbidas" en el lenguaje puramente matemático en el que se pretende expresar la teoría. Con respecto al contenido empírico y observacional, Tegmark insiste en que las observaciones particulares involucradas en la formulación y contrastación de una teoría pueden ser tratadas como cualidades meramente intersubjetivas, un aspecto más del mundo físico que puede por tanto ser pasible de una descripción puramente matemática. Su caso respecto de las condiciones iniciales es más débil. Por hipótesis, la TdT es una descripción completa del universo, lo que no deja lugar a condiciones iniciales de ningún tipo por fuera de ella, y si así lo hiciere, entonces no es una TdT (TEGMARK, 2008, p. 117). Por lo tanto, según Tegmark, una TdT no debe, consistentemente con su carácter, contemplar condiciones iniciales (2008, p. 117). Este escenario presenta múltiples dificultades. La aplicación de cualquier ley, al menos de acuerdo a consideraciones metacientíficas universalmente aceptadas, requiere de la apelación a condiciones iniciales. Del mismo modo, la explicación científica de eventos singulares, sea invocando leyes, mecanismos o causas parece por principio imposible si no se toman en cuenta las circunstancias particulares que determinan el fenómeno a explicar, es decir, sus condiciones iniciales. Más en general, resulta muy difícil imaginar cómo podrían darse cuenta del contenido empírico de las teorías en ausencia de condiciones iniciales.

Una segunda preocupación se vincula con las capacidades representativas del lenguaje matemático. Como es sabido, una misma descripción matemática puede aplicarse de modo igualmente efectivo a dos sistemas físicamente diferentes (e.g. osciladores armónicos e inarmónicos). Esta es, de hecho, una de las tantas caras que toma el problema de la relatividad ontológica señalado por Quine. La mera posibilidad de establecer una diferencia física entre los dos sistemas da cuenta del hecho de que hay algo que la representación matemática no puede capturar, por lo que ella es, por tanto, incompleta. Si bien Tegmark

Manuscrito - Rev. Int. Fil. Campinas, v. 40, n. 2, pp. 71-97, abr.jun. 2017. 
no brinda una respuesta a este problema, podría pensarse que un modo posible de eludirlo sería simplemente desconocerlo como tal. Podría argüirse que la posibilidad de describir dos sistemas por con un mismo puñado de ecuaciones se restringe a niveles relativamente simples de complejidad, una vez que uno escarba en niveles más profundos esa posibilidad desaparece y la descripción matemática ve su completitud restaurada.

Volviendo al argumento, la TdT expresada en lenguaje puramente matemático está libre de todo "bagaje" humano. La jugada clave de Tegmark es mostrar que esta idea, en conjunción con la HRE, implica lo que denomina la Hipótesis del Universo Matemático (HUM):

Mientras que la terminología habitual en los libros de texto de física es que la realidad externa es descrita por las matemáticas, la HUM afirma que [la realidad] es matemática (más específicamente, una estructura matemática). Esto corresponde a la versión "óntica" del realismo estructural. (TEGMARK, 2008, p. 107)

El modo en que la HRE y una eventual TdT matemáticamente expresada implican la HUM es extremadamente simple: la TdT es una estructura matemática; y si constituye una descripción completa de la realidad, ello significa que es isomórfica con la realidad. Ahora bien, dos estructuras isomórficas son idénticas, por lo tanto, la realidad es una estructura matemática (TEGMARK, 2008, p. 107).

Nótese que no cabe aquí argüir que podría haber un sentido de representación o descripción diferente al del mero isomorfismo: todo vínculo de representación entre un modelo matemático y un sistema target que trascienda el isomorfismo estructural requeriría de algún tipo de interpretación de ese modelo, lo que por principio ha sido eliminado con el resto del bagaje humano que representan los significados. Tampoco puede aducirse que ello acaba con una teoría menos completa ya que, por hipótesis, TdT es completa. Sostengo que hay, no obstante, al menos dos maneras de atacar este argumento, la primera tiende a señalar algunos problemas conceptuales en su formulación, la segunda le imputa una petición principio.

Comencemos por los problemas conceptuales. El argumento nos pide que pongamos en juego una serie de distinciones. Una de ellas es, por

Manuscrito - Rev. Int. Fil. Campinas, v. 40, n. 2, pp. 71-97, abr.jun. 2017. 
supuesto, la distinción entre entidades de naturaleza física y matemática. La segunda se desprende de la misma noción de "descripción" o "representación" completa de la realidad. Hay algo (en este caso un lenguaje matemático) que representa otra cosa (la realidad física externa). Por último, aquello que es representado, a saber, la realidad, es calificado como "externo". Como hemos visto, no puede tratarse de algo externo a nuestras representaciones o contenidos de observación, ya que estos son parte de la realidad matemáticamente descrita por la TdT. La opción obvia parece ser entonces que esa realidad es externa a la TdT o al dispositivo mediante el que la TdT la describe (que, según Tegmark, resultan ser la misma cosa). Ahora bien, estas tres distinciones necesarias para comenzar el argumento son arrojadas por la borda una vez que éste está en marcha. O peor aún, son negadas a la hora de formular la conclusión. Ello parece poner en jaque la propia posibilidad de formular un argumento como este. La realidad "externa" se confunde ahora con lo que pretende representarla, la mera idea de "descripción" se diluye en el mismo movimiento a menos que se tome como válida la posibilidad de que algo se describa o represente a sí mismo. Todavía más preocupante es el caso de la distinción entre realidad física y matemática, ¿cómo es posible mantener de modo significativo una distinción si se postula una identidad entre ambas? Podría pensarse que la pretendida identidad enmascara una suerte de emergencia de la realidad física de una estructura matemática más fundamental. Pero ese no es el caso dados los términos del argumento, y si lo fuera, se nos estaría debiendo un relato acerca de cómo la existencia de entidades físicas puede ser causada por entidades matemáticas.

Mi segunda objeción se dirige a un aspecto puntual del argumento, concretamente a la inferencia de la HUM a partir de la idea de que la TdT y la realidad son isomórficas. Dicha afirmación podría interpretarse en más de un sentido. En la literatura acerca de modelos científicos es harto común afirmar que una estructura resulta isomórfica con los fenómenos, o con parte de un sistema target considerado en alguno de sus aspectos. Lo que en esos casos quiere en realidad afirmarse (y lo que de hecho se afirma en contextos en los que la cuestión de la representación está en foco) es que la estructura matemática resulta isomórfica, no con los fenómenos mismos, sino con otra estructura que es posible construir por

Manuscrito - Rev. Int. Fil. Campinas, v. 40, n. 2, pp. 71-97, abr.jun. 2017. 
abstracción a partir de ciertas característica natural o pragmáticamente relevantes del sistema target. Este sentido laxo de isomorfismo, sin embargo, no es el empleado en el argumento. Por el contrario, se da por sentado que una relación de isomorfismo sólo puede ocurrir entre estructuras matemáticas idénticas. Es decir, el sentido de isomorfismo considerado aquí es fuerte, lo que implica que se trata de una relación que sólo puede darse entre dos estructuras abstractas. Pero si ese es el caso, la premisa que afirma que la realidad externa es isomórfica con la TdT no puede ser usada para derivar HUM, puesto que HUM afirma que la realidad externa es una estructura matemática, y la premisa en cuestión presupone que la realidad externa es una estructura matemática (puesto que, de no ser así, no había modo en que pudiera ser isomórfica respecto de otra estructura matemática, como lo es la TdT). El argumento incurre así en una flagrante petición de principio.

Si bien otras aristas de la argumentación de Tegmark presentan problemas análogos, considero que los que acabo de mostrar resultan suficientes para desestimar su posición.

\section{Realismo Estructural Óntico, leyes y estructuras físicas}

La preocupación de que el compromiso ontológico del REO colapse en el platonismo fue una de las más acuciantes desde sus inicios. Quién más atención le prestó a ese punto fue Cao, para él la apuesta del REO consiste en un "intento de disolver las entidades físicas en estructuras matemáticas", por lo que sus defensores se desplazan "del realismo científico al idealismo platónico" (2003, p. 57). Esta imputación dio lugar a una extensa discusión con French \& Ladyman, quienes nunca resignaron el carácter físico de las estructuras.

¿Qué es lo que hace que una estructura sea 'física'? Bueno, crudamente, que puede estar relacionada — en nuestro marco, a través de isomorfismos parciales- con los 'fenómenos' (físicos). Así es como el 'contenido físico' entra. Menos trivialmente, lo matemático se puede distinguir de lo físico en que este último es además causal. (FRENCH \& LADYMAN, 2003, p. 75).

Manuscrito - Rev. Int. Fil. Campinas, v. 40, n. 2, pp. 71-97, abr.jun. 2017. 
En este estadio de la discusión son entonces dos los rasgos que determinan el carácter físico de las estructuras, su relación con los fenómenos físicos y su carácter causal. Sólo el último será conservado en defensas posteriores, y no resulta difícil ver por qué. Cualquier forma más o menos sofisticada de antirrealismo científico -el Empirismo Constructivo de van Fraassen es un obvio ejemplo- admite que el conocimiento científico es acumulativo en cuanto a la estructura de los fenómenos físicos. Pero ello no significa, por supuesto, que todas las teorías físicas sean empíricas en el sentido de limitarse a contabilizar o generalizar sobre observaciones. Los fenómenos, en la forma de modelos de datos, se relacionan con los modelos teóricos por vía de sus subestructuras empíricas. En otras palabras, no hace falta ser realista para afirmar que las estructuras matemáticas con las que una teoría "describe" el mundo inobservable se relacionan con los fenómenos (a través de isomorfismos, isomorfismos parciales, similaridad o algún vínculo análogo). Mucho menos es el caso que la posibilidad de relacionar una estructura matemática con un dominio de fenómenos físicos haga de la estructura matemática una estructura física.

$\mathrm{El}$ argumento que sobrevive hasta formulaciones recientes del REO es el que afirma el carácter causal de las estructuras o, como se lo suele explicitar, su naturaleza modal. Éste aparece en French (2014) — junto con el trabajo de Ladyman \& Ross (2007), la defensa más completa del REO publicada hasta la fecha- conjuntamente con un segundo argumento. Sumariamente, French hace uso de una distinción entre la representación de la estructura y la presentación de los objetos que aparecen en ella por medio de sus estructuras compartidas. Según entiende, si bien la representación hace uso de conceptos abstractos, eso no significa que la estructura compartida por los objetos postulados por la teoría no pueda ser presentada como ontológicamente primaria.

El primer argumento afirma, entonces, que el carácter físico de las estructuras postuladas por el REO está dado por su naturaleza modal, rasgo ausente en las estructuras matemáticas. Sumariamente, French sostiene que debe darse un carácter primitivo a las leyes naturales por sobre las propiedades y los objetos. Ahora bien, esas leyes parecen no ser concebidas al modo del realismo nomológico tradicional, aquí "las leyes

Manuscrito - Rev. Int. Fil. Campinas, v. 40, n. 2, pp. 71-97, abr.jun. 2017. 
son simplemente aspectos relevantes de la estructura subyacente" (FRENCH, 2014, p. 302). La noción de "gobierno" es sólo una metáfora para expresar primacía ontológica. De ese modo, la naturaleza nomológica y por ende modal de las regularidades está asegurada de antemano. Sin embargo, sostengo, esa caracterización diluye la distinción entre leyes y generalizaciones accidentales, por lo que la naturaleza "nomológica" de las estructuras no está justificada.

Pero hay una razón adicional por la que esta versión del REO fracasa en justificar el carácter físico de las estructuras por vía de la modalidad. Esta razón alcanza también a la mayoría de las variantes no-eliminativistas que utilizan el mismo recurso a idénticos fines. Brevemente, mi argumento es el siguiente: la modalidad puede usarse para distinguir lo físico de lo matemático, bajo la asunción de que lo matemático no podría ser causalmente eficaz. Pero la mera eficacia causal no es suficiente para determinar el carácter físico de una entidad, puesto que las entidades mentales también han sido juzgadas como causalmente eficaces, y no por ello como físicas. En suma, la eficacia causal no es condición suficiente para la atribución de carácter físico.

Ocupémonos ahora del segundo argumento, que podría reconstruirse del siguiente modo: la ciencia se ocupa de describir fenómenos; la física cuántica, concretamente, se interesa por cierto tipo de fenómenos físicos y los describe como estructuras en el marco de la teoría de grupos. Por su parte, la filosofía de la ciencia se ocupa de describir teorías y (si al menos cierta dosis de realismo es admitida en su ejercicio) la relación que esas teorías guardan con el mundo. En el marco de la versión de la concepción semántica abrazada por French, la herramienta para efectuar esa descripción son las estructuras parciales expresadas en el leguaje de la teoría de conjuntos. En el nivel científico se ofrecen estructuras matemáticas que describen los fenómenos, y en el nivel metacientífico estructuras parciales que reflejan la relación entre los modelos teóricos, los modelos de datos y la estructura del mundo (FRENCH, 2014, p. 115). French vincula estos dos niveles con la distinción introducida por Brading \& Landry (2006) entre presentación y representación. Según su interpretación, debe distinguirse "la presentación de las entidades putativas por medio de la estructura compartida dada por nuestras teorías, y la representación de tales objetos (como aspectos del mundo) por dichas teorías. La pregunta obvia

Manuscrito - Rev. Int. Fil. Campinas, v. 40, n. 2, pp. 71-97, abr.jun. 2017. 
entonces es: ¿cómo es representada esa estructura compartida?” (2014, p. 101, el énfasis me pertenece). La respuesta de French, como ya he sugerido, es que dicha estructura compartida es representada en el nivel matateórico por medio de estructuras parciales. Estos dos niveles son cruciales para trazar una frontera entre lo matemático y lo físico en lo concerniente a las estructuras, porque es precisamente en el nivel de la representación metateórica donde se revela la naturaleza física de las estructuras que son meramente presentadas por la teoría mediante una descripción puramente matemática (en el caso de la física cuántica, por medio de simetrías e invariantes expresadas mediante teoría de grupos):

Insistimos en que la estructura física es concreta, ya que puede estar relacionada - a través de isomorfismos parciales en el marco de las estructuras parciales, por ejemplo-, con los 'fenómenos' (físicos). Es así como el 'contenido físico' entra en nuestras teorías y les permite ser (al menos parcialmente) interpretadas. (...) Pero claramente ese contenido debe ser entendido como fundamentalmente no-matemático (FRENCH, 2014, p. 199)

Sostengo que hay al menos tres sentidos en los que la interpretación que French hace de la distinción defendida por Brading \& Landry es fallida (y lo suficientemente fallida para no poder sostenerse como una variación alternativa de dicha distinción), lo cual socava su argumento a favor de la naturaleza física de las estructuras.

Previo a especificar esos tres aspectos problemáticos, conviene echar un vistazo a la distinción tal como Brading \& Landry la formulan. En tal sentido cabe destacar que su objetivo es doble. Por una parte, buscan dar con el punto preciso en el que el estructuralismo matemático se diferencia del estructuralismo científico; por otra, sientan las bases para una clasificación de grados de compromiso ontológico dentro del estructuralismo científico. En cuanto al primer punto, la distinción entre presentar y representar es clave. En una teoría matemática presentar un objeto mostrando su lugar en una estructura es equivalente a representarlo; en otras palabras, basta que una teoría hable de una entidad matemática para que dicha entidad sea el objeto de ese discurso. En cambio, una teoría física puede hablar acerca de electrones (o unicornios), sin ser acerca de electrones

Manuscrito - Rev. Int. Fil. Campinas, v. 40, n. 2, pp. 71-97, abr.jun. 2017. 
(unicornios), presentarlos no es aquí equivalente a representarlos exitosamente o traerlos a la existencia (BRADING \& LANDRY, 2006, p. 573).

Ahora bien, ya en el dominio del estructuralismo científico, uno es libre de adoptar distintos grados de compromiso respecto de la capacidad representativa de las teorías adoptando una actitud (sance) metodológica, empírica o realista. Es crucial aquí dar cuenta de cómo ocurre dicha decisión y cuál es exactamente su objeto para acabar de entender cabalmente la distinción entre presentar y representar. La teoría presenta una clase de objetos en sus modelos teóricos, esos modelos resultan aplicables a un modelo de datos si ambos tienen una estructura compartida, esto es, si puede probarse alguna relación relevante entre ellos (isomorfismo, homomorfismo, isomorfismo parcial, etc.). Pero esto es sólo un primer paso que no involucra todavía un contacto con el mundo. Dicho contacto se produce solamente si se brinda un relato adecuado de cómo esos modelos de datos efectivamente representan los fenómenos, en otras palabras, debemos "dar cuenta de la representación en términos de la estructura compartida entre los modelos de datos y los fenómenos, de manera que los fenómenos acerca de los que la teoría es tengan la estructura apropiada" (BRADING \& LANDRY, 2006, p. 575). No es otra cosa que el problema de la representación sobre el que Reichenbach ([1920] 1965) llamó la atención, y que van Fraassen resume magistralmente: “¿cómo puede una entidad abstracta, como un espacio matemático, representar algo que no es abstracto, algo en la naturaleza?" (VAN FRAASSEN, 2006b, p. 537). El tipo de respuesta que se ofrezca depende del grado de compromiso ontológico que se esté dispuesto a asumir. Desde una actitud realista, es el mundo mismo el que estructura los fenómenos, y un modelo de datos los representa si se acomoda a esa estructura. Desde una actitud empirista (como la que van Fraassen adopta), es la teoría la que proyecta una estructura en los fenómenos ${ }^{3}$. Desde la actitud metodológica que Brading \& Landry defienden, en cambio, el problema es simplemente ignorado,

\footnotetext{
${ }^{3}$ Si bien este es el modo en que Brading \& Landry caracterizan la actitud empirista, podría pensarse que para ser consecuente con su tradición, un empirista debería afirmar que, aunque construido, el modelo de datos captura algún aspecto presente en los fenómenos.
}

Manuscrito - Rev. Int. Fil. Campinas, v. 40, n. 2, pp. 71-97, abr.jun. 2017. 
concentrándose sólo en las relaciones entre el modelo de datos y las subestructuras empíricas de los modelos teóricos.

Con esta breve reconstrucción en mente puede verse con más claridad cuáles son los tres sentidos en los que afirmo que French malinterpreta la distinción introducida por Brading \& Landry.

El primero de ellos se vincula con el rol del enfoque de estructuras parciales (y de la concepción semántica de los teorías en general) en la representación de la realidad. Una de las principales motivaciones para trazar una distinción como la que Brading \& Landry proponen consiste en mostrar las limitaciones propias de las herramientas de la concepción semántica para brindar, por sí solas, un relato completo de cómo las teorías se relacionan con el mundo. Las herramientas semánticas requieren de una propuesta ontológica que dé cuenta de cómo los modelos de datos capturan la estructura objetiva de los fenómenos (con una estrategia semejante esa misma idea fue defendida por Frigg, 2006). En tal sentido lo que French toma por el momento de la representación en el lenguaje metateórico es sólo un fragmento de lo realmente se requiere. Él mismo parece reconocer implícitamente eso al afirmar que la noción de isomorfismo parcial propia de ese marco se extiende "tanto 'verticalmente' como 'horizontalmente' (...) [para] capturar las relaciones entre teóricas y las relaciones con los modelos de datos" (FRENCH, 2014, p. 104). Es decir: excluye la relación con los fenómenos en sí mismos.

En segundo lugar, contrariamente a lo que se afirma en el argumento, en la distinción de Brading \& Landry no hay un desdoblamiento en dos niveles de representación. Además, es preciso remarcar que, para ellas, lo que en última instancia se representa son los fenómenos, no una estructura compartida. En varias ocasiones (tal como en la cita consignada más arriba) French insiste en que "la 'estructura compartida' puede ser representada por estructuras modelo-teóricas (parciales), más iso/homomorfismos (parciales) asociados" (2014, p. 105, énfasis añadido). Hay allí, para comenzar, un problema con el sentido de 'representación'. En el modo que Brading \& Landry lo emplean, en el marco del estructuralismo científico una estructura compartida no puede representarse, puesto que no es algo en el mundo, sino una relación entre el mundo y la teoría (independientemente, por supuesto, de que la ontología del mundo se esclarezca en términos de una metafísica tradicional o estructuralista). Por

Manuscrito - Rev. Int. Fil. Campinas, v. 40, n. 2, pp. 71-97, abr.jun. 2017. 
otra parte, y aun suponiendo que French pudiera estar adaptando la noción a sus propósitos, la relación de compartir estructura con es, en sentido estricto, sólo aplicable cuando ambos términos son estructuras matemáticas. Y en sentido laxo (como una relación entre la teoría y los fenómenos), secundaria, puesto que es una mera consecuencia de la afirmación de que los fenómenos tienen estructura "natural" o "dada".

En tercer lugar, incluso si se diese por válido ese sentido laxo de estructura compartida, éste no nos informa por sí mismo nada acerca de la naturaleza de las entidades (objetos, propiedades, estructuras, etc.) reales. Pero además, y más importante, si lo que efectivamente se representa, tal como French insiste en remarcar, es la estructura compartida entre los fenómenos y el modelo de datos, ello parece abonar desde todo punto de vista la conclusión de que las estructuras del REO son entidades abstractas más que de naturaleza física. El compartir estructura es una relación que se da solamente entre entidades matemáticas y, en el mejor de los casos, entre una entidad matemática (el modelo de datos) y una estructura concreta determinada por una función que capture las relaciones "relevantes" o "naturales" entre los fenómenos, pero la estructura compartida, qua relación, no puede ser sino una entidad abstracta producto de poner en correlación fenómenos y modelo de datos.

Estas tres razones muestran que la estrategia de French para justificar el carácter físico de las estructuras postuladas por el REO mediante la distinción entre presentación y representación es fallida.

\section{4. ¿Qué es lo físico, después de todo?}

El problema de cómo dar cuenta del carácter físico de las entidades básicas aparece como especialmente acuciante en una propuesta ontológica tan radical como la del REO, pero brindar una noción aceptable de entidad física no es un asunto menos problemático en el marco de las disputas metafísicas sobre el fisicalismo. Explorar este punto no sólo puede resultar iluminador para la cuestión central de este trabajo, sino para dar cuenta de su rol en los debates sobre el realismo científico. En efecto, en ellos está presupuesta una noción folk de entidad física, y si bien no hay en general compromisos explícitos, existe una actitud fisicalista en cuanto

Manuscrito - Rev. Int. Fil. Campinas, v. 40, n. 2, pp. 71-97, abr.-jun. 2017. 
al tipo de entidades de las que hablan las teorías y en cuanto a los compromisos realistas que deberíamos asumir respecto de ellas. Ahora bien, más allá de ello, el realismo científico no requiere del fisicalismo ni lo implica. Afirmar que las entidades postuladas por nuestras mejores teorías científicas existen no implica, ni que ellas sean físicas, ni que, si efectivamente son físicas, ellas sean lo único que existe o la única categoría ontológica con la que uno debería comprometerse. Por ejemplo, el realismo científico es absolutamente compatible con un teísmo, o con un dualismo fuerte respecto de la mente. Esto se vincula con el hecho de que a la hora de definir los compromisos realistas se hace una referencia ineludible a objetos, y esos objetos son en general objetos físicos. Es por ello que la cuestión del fisicalismo no está tematizada y se toma usualmente como un presupuesto. Ahora bien, en el caso del REO, esto pasa a tener un sentido especial y preocupante, puesto que además de defender la primacía ontológica de las estructuras por sobre entidades de otro tipo, casi todos los defensores del REO insisten en que esas estructuras no son estructuras matemáticas o abstractas, sino estructuras físicas. Por tanto, la necesidad de definir una noción de entidad física tal que pueda predicarse de una estructura es central. Entonces, si bien el REO, como toda forma de realismo científico, resulta totalmente independiente de la posición que uno asuma respecto del fisicalismo y, previamente, de la noción de entidad física que se adopte, tanto el fisicalista como quien sostenga el REO precisan de una noción clara, precisa y funcional de entidad física. El primero para decir que todo lo que hay cumple con esa condición. El segundo para decir que la categoría ontológica fundamental que postula en términos puramente estructurales es de hecho una categoría física, y que esas estructuras objetivas de las que el REO habla son efectivamente estructuras físicas. La noción que voy a intentar delinear puede funcionar como una herramienta para dar cuenta de ambas cosas. Por una parte, y principalmente, de una noción de entidad física que se acomode al dictum del REO, que afirma que las entidades básicas son estructuras físicas, por otra parte, a las necesidades que el fisicalista puede tener para afirmar que su posición es verdadera partiendo de una noción aceptable y funcional de entidad física.

Hay dos grandes modos de caracterizar la noción de entidad física, o bien a priori, en una reflexión filosófica (que denominaré enfoque por

Manuscrito - Rev. Int. Fil. Campinas, v. 40, n. 2, pp. 71-97, abr.-jun. 2017. 
objeto), o bien a posteriori, teniendo en cuenta lo que nuestras teorías físicas postulan como entidades reales (enfoque por teoría). Según entiendo, ninguna resulta adecuada a nuestros fines, por lo que propongo un enfoque alternativo.

\subsection{El enfoque por objeto}

El fisicalismo es heredero del materialismo, una tradición filosófica mucho más antigua cuya piedra fundamental es la idea de que la única sustancia que existe es la sustancia material. Esta tesis, que podría identificarse como el componente positivo del materialismo, está acompañada de un componente negativo que consiste en negar la existencia de una sustancia espiritual o mental. Aunque tal vez en distinta medida, ambos sobreviven en el fisicalismo contemporáneo. El componente positivo persiste en la idea de que hay una suerte de noción folk de entidad física que es la fuente de nuestras intuiciones acerca de lo que contaría en última instancia como físico. En tal sentido una propiedad es física si corresponde a una de las características de un objeto intuitivamente físico (STOLJAR, 2001, p. 54). No caben dudas de que entendemos en general lo que una entidad física es, ni de que esa comprensión es la fuente de muchas de nuestras creencias en torno al fisicalismo. Sin embargo, esa supervivencia al nivel de las intuiciones se desintegra en un análisis más cuidadoso, en especial, considerada a la luz de algunos resultados de la ciencia moderna. En efecto, nuestras mejores teorías acerca del mundo físico postulan entidades fundamentales que tienen pocas o ninguna de las características que habitualmente le adscribimos a los objetos intuitivamente físicos. Y eso llega a incluir entidades como el espacio y el tiempo, que no sólo son conflictivas en sí mismas respecto de su carácter físico, sino que usualmente resultan parte del aparato conceptual que usamos para atribuirle ese carácter a otras entidades.

El componente negativo del materialismo, en cambio, sobrevive en el fisicalismo tanto en el nivel de las intuiciones como del análisis filosófico. Su forma más común de expresión, que constituye el núcleo de lo que denomino enfoque por objeto, es la definición de lo físico por su carácter no mental. Esto se enmarca, a su vez, en la convicción más general de que la tesis fisicalista no es una hipótesis científica, ni una que primariamente

Manuscrito - Rev. Int. Fil. Campinas, v. 40, n. 2, pp. 71-97, abr.jun. 2017. 
pueda decidirse por medios empíricos. En su defensa canónica del enfoque por objeto Crook \& Gillett afirman que el fisicalismo debe regresar a las intuiciones fundantes del materialismo para "ubicarse una vez más como como una hipótesis filosófica basada sobre conceptos desarrollados para enfrentar problemas metafísicos" (2001, p. 336). Es por ello que el rasgo de no mentalidad es un requisito fundamental, que luego incorporarán a su definición de la tesis fisicalista, en sus palabras: "las entidades mentales no están entre las entidades lógicamente contingentes que componen todas las demás entidades lógicamente contingentes" (2001, p. 349). Del mismo modo, Wilson (2006) (quien defiende un enfoque mixto en algunos sentidos próximo al que este trabajo propone) afirma que el requisito de No-Mentalidad Fundamental (NMF) es indispensable en una caracterización adecuada de lo físico. En pocas palabras, una entidad es física si es objeto de tratamiento de la física fundamental (actual o futura) y no es fudamentalmente mental (WILSON, 2006, p. 72).

El requisito de NMF es, en sus distintas presentaciones, el punto central del enfoque por objeto, tanto en las versiones más radicales que como la de Crook \& Gillett - toman la reflexión filosófica como el único camino para caracterizar lo físico, como en las que reconocen un papel relevante e incluso esencial a la ciencia.

La mayor objeción a la inclusión del requisito de NMF en la caracterización de lo físico se asocia a la posibilidad de que se trate de una demanda ad hoc, vinculada más a preocupaciones propias de la filosofía de la mente que a la tarea de brindar condiciones suficientes y necesarias para afirmar que una entidad es física. En términos de Wilson, se trata del problema de la no-sistematicidad. Es claro que la existencia de entidades fundamentalmente mentales parece refutar el fisicalismo, pero "intuitivamente, el fisicalismo sería también refutado si descubriésemos que entidades correspondientes a órdenes de complejidad constitucional relativamente bajo son agentes morales o actúan libremente, o que en ciertas respuestas estéticas están involucradas una nueva interacción o fuerza fundamental". Es por ello que pareciera que al requisito de NMF debieran adicionarse otros de "no moralidad fundamental, no libre albedrio fundamental, no estética fundamental" (WILSON, 2006, p. 74-75). En suma, la no-sistematicidad implica que la caracterización de lo físico no consta de

Manuscrito - Rev. Int. Fil. Campinas, v. 40, n. 2, pp. 71-97, abr.jun. 2017. 
condiciones necesarias y suficientes sino de un conjunto de rasgos que se incorporan a medida que posibles "falsadores potenciales" de la tesis fisicalista van siendo invocados.

La respuesta de Wilson en defensa del requisito de NMF es que la nosistematicidad es en realidad aparente:

Es plausible que la mayoría, y quizás todas las entidades cuya postulación como entidades fundamentales falsaría intuitivamente el fisicalismo, y cuya postulación como entidades fundamentales continúa siendo una posibilidad real, tienen en común que la mentalidad es una condición previa para su existencia en un sentido fuerte, de acuerdo con el cual su existencia está en cierto grado constituida por la mentalidad. (WILSON, 2006, p. 76)

Si bien creo que esta defensa es inadecuada, argumentar de modo completo contra esa respuesta requeriría relevar y clarificar una serie de cuestiones previas que en pos de la simplicidad han sido relegadas en esta exposición. Pero bastará dar cuenta aquí de que las falencias de este argumento muestran que el enfoque por objeto resulta inconveniente para una caracterización del fisicalismo coherente con los propósitos del REO. Ello, lateralmente, me permite sugerir que el requisito de NMF no puede resultar en general defendible como parte de una caracterización de lo físico. Mi punto es simple: las entidades abstractas (e.g., las entidades matemáticas) no tienen (al menos según una doctrina filosófica que goza de cierta popularidad) la mentalidad como una condición previa para su existencia $y$, sin embargo, pueden ser postuladas como entidades fundamentales, incluso físicamente fundamentales.

No es extraño que Wilson no haya tenido en mente esta posibilidad. Las entidades abstractas se encuentran en el campo del análisis metafísico de la tesis fisicalista al margen de la discusión, donde nadie les presta demasiada atención. Y hay buenas razones para que ese sea el caso. Primero, su mismo nombre parece indicar que ellas no son más que abstracciones a partir de entidades que las preceden en nivel de fundamentalidad, por lo que no parecen constituir un riesgo real para la tesis. Segundo, incluso si este no fuera el caso y entidades como números, clases o estructuras gozaran de una existencia independiente y de un grado de realidad equiparable al de las entidades fundamentales del mundo físico,

Manuscrito - Rev. Int. Fil. Campinas, v. 40, n. 2, pp. 71-97, abr.jun. 2017. 
se asume tácitamente en la discusión que ambas clases de entidades pertenecen a niveles diferentes de la realidad y que las entidades abstractas, de existir, no pueden reclamar un puesto entre las que resultan fundamentales en la constitución del mundo físico. Ambas razones, sin embargo, podrían quedar socavadas en el marco del REO. Al menos en la versión de Tegmark, tanto la independencia ontológica como la segregación categorial de lo matemático y lo físico quedan impugnadas por la HUM. Y si se atiende a las razones que he esbozado en la sección precedente, lo propio podría afirmarse de la versión de French, en tanto ésta falla en brindar una distinción convincente entre las estructuras matemáticas y las físicas.

Claro que los problemas propios de la peculiar concepción de Tegmark podrían ser invocados por Wilson como una razón para sostener que el requisito de NMF no queda efectivamente puesto en jaque por la posibilidad de postular entidades abstractas como físicamente fundamentales. Pero si bien esto puede relativizar la importancia de esta posibilidad en los debates sobre el fisicalismo, es claro que en el contexto de formulación del REO se hace relevante. El componente central del enfoque por objeto, el requisito de NMF, no resulta suficiente para dar cuenta de modo convincente del carácter físico de las estructuras que el REO postula como fundamentales. De hecho el REO aparece como una amenaza global para dicho enfoque, por lo que mal podría ser este último parte de su marco.

Por otra parte, y tal vez más importante aún, lo que fuere que haya sobrevivido del componente positivo del materialismo en el fisicalismo se ve igualmente socavado por los presupuestos metafísicos del REO. Si se admite que sus estructuras son efectivamente físicas, esto ha de hacerse a pesar de que poseen pocas o ninguna de las características habitualmente e intuitivamente asociadas a las entidades físicas.

En resuenen, el enfoque que denomino por objeto no resulta el marco apropiado para caracterizar una noción de estructura física como la que el REO requiere. De hecho, la propia articulación del REO se opone tanto al requisito de NMF como a la posibilidad de atar la noción de objeto a propiedades de los objetos del sentido común.

Manuscrito - Rev. Int. Fil. Campinas, v. 40, n. 2, pp. 71-97, abr.jun. 2017. 


\subsection{El enfoque por teoría}

Las dificultades propias del enfoque por objeto, vinculadas en su mayoría a sus resabios materialistas, han hecho que buena parte de los filósofos involucrados en el debate se inclinasen por la idea de que es la física en última instancia la que ha de determinar el contenido de la tesis fisicalista. Dado que sus ataques a la metafísica tradicional se ven impulsados por los resultados de esa ciencia, no es extraño que varios de los promotores del REO se identifiquen con esta corriente.

De este modo, el enfoque por teoría señala, en pocas palabas, que una entidad es física si es objeto de tratamiento de la física. Sus problemas son bien conocidos y de larga data. El más relevante de ellos es sin dudas el llamado Dilema de Hempel (1969; 1980), que señala, en resumidas cuentas, que la tesis fisicalista caracterizada por este enfoque es trivial o falsa. El primer cuerno del dilema considera la posibilidad de que se tome en cuenta la física tal como se ha desarrollado hasta el momento actual. Si ese es el caso, dado que la física actual es casi sin dudas incompleta e imprecisa, la tesis fisicalista resultaría falsa. El segundo cuerno contempla en cambio la posibilidad de que el contenido de la tesis fisicalista se fije con referencia a una física futura o ideal, y en tal sentido la tesis resultaría trivialmente verdadera. Después de todo, una física futura o ideal tratará acerca de algunas entidades, y si esas entidades son las entidades físicas simplemente por hipótesis, no hay circunstancia posible en la que el fisicalismo pudiera resultar falso.

Hay varias estrategias para defender el enfoque por teoría del Dilema de Hempel. Entre las que cuestionan el primer cuerno, usualmente denominadas presentistas, la más notoria es sin dudas la de Melnyk (1997). Según entiende, el hecho de que el fisicalismo descanse en la física actual, y por lo tanto tenga grandes chances de ser falso, no es en sí mismo un problema. Los realistas científicos se encuentran en la misma situación respecto de las teorías y no por ello resignan el realismo. Es así que asumir la actitud de los realistas (lo que denomina "actitud RC") es el camino a seguir.

[D]ado que un fisicalista es simplemente alguien que adopta la actitud RC hacia el fisicalismo, el mero hecho de que la historia de la ciencia física haga que sea poco probable que el fisicalismo sea Manuscrito - Rev. Int. Fil. Campinas, v. 40, n. 2, pp. 71-97, abr.jun. 2017. 
cierto no ofrece ninguna razón por sí mismo para dejar de ser fisicalista; uno puede seguir siendo fisicalista, con tal de que el fisicalismo, aunque resulte poco probable, sea más probable que sus rivales relevantes. (MELNYK, 1997, p. 632)

Múltiples críticas se han realizado contra este argumento, pero me permito agregar dos objeciones propias que considero suficientes para descartarlo. Primero, en el debate contemporáneo, toda forma de realismo científico más o menos sólida asume como punto de partida que las teorías son en sentido estricto falsas si se las considera globalmente. El realismo científico actual toma la forma de un realismo selectivo: mediante diferentes estrategias se da cuenta de cuáles son las partes o aspectos de una teoría que resultan responsables de su éxito predictivo, y es sólo respecto de ellas que el compromiso realista se cristaliza. Además, incluso así concebido, el compromiso con el realismo no implica que el antirrealismo sea menos probablemente verdadero. Por el contrario es ampliamente reconocido que realismo y antirrealismo científicos son actitudes respecto del estatus epistémico de las teorías que, si bien pueden ser apuntaladas mediante argumentos, no pueden juzgarse en función de su probabilidad de verdad. La actitud RC, por tanto, no se parece mucho a la actitud que efectivamente asumen los realistas científicos. Pero hay una segunda razón: si el realismo científico es un punto de partida necesario para el sustento del fisicalismo, eso implica que un antirrealista, si quisiese abrazar el fisicalismo, estaría adoptando una tesis que no puede sustentar. Y eso es claramente absurdo: intuitivamente resulta totalmente compatible sostener que las entidades fundamentales de la realidad son físicas y, al mismo tiempo, abstenerse de un compromiso ontológico con las entidades inobservables postuladas por la ciencia. Este argumento implica por tanto una tesis por lo menos implausible, a saber, que el fisicalismo es incompatible con el antirrealismo científico.

Los defensores del REO, en cambio, optan por el futurismo, i.e., por la estrategia que busca eludir el dilema hempeliano atacando la conclusión del segundo cuerno. Brown \& Ladyman abrazan explícitamente este enfoque, aunque son conscientes de los problemas que conlleva su forma pura:

Manuscrito - Rev. Int. Fil. Campinas, v. 40, n. 2, pp. 71-97, abr.-jun. 2017. 
Muchos físicos y filósofos consideran que es analíticamente verdadero que la física debe ser capaz en principio de dar cuenta de todo fenómeno natural. Por ejemplo, los enlaces químicos no son, estrictamente hablando, parte de la física fundamental, pero no dejan de ser explicados por ella en términos de fuerzas electrostáticas entre partículas cargadas. Por lo tanto, puesto que los fenómenos mentales son fenómenos sin duda naturales, es analítico en esta definición de la física que una física fundamental futura y completa será capaz de dar cuenta de lo mental (...). De esta mirada acerca de lo que la física es se sigue que si es necesario cuantificar sobre ectoplasma para dar cuenta de un fenómeno natural, entonces el comportamiento del ectoplasma está en el ámbito de la física. Si se adopta esta perspectiva, se deduce que si el fisicalismo se caracteriza exclusivamente en términos del contenido de la física futura, entonces es trivialmente verdadero. (BROWN \& LADYMAN, 2009, p. 22)

La solución que proponen está en línea con la ofrecida por Wilson, aunque, dado que no quieren formular un enfoque abiertamente mixto, codifican el requisito de NMF en la forma de una predicción. Así, la tesis fisicalista debe complementarse con la "predicción de que la física fundamental no postulará entidades que posean esencialmente características mentales" (BROWN \& LADYMAN, 2009, p. 24). Es claro por qué esta propuesta es ventajosa para quien defiende el REO. Si se acepta que la mejor interpretación de la ciencia moderna requiere postular estructuras como las entidades fundamentales, ellas son físicas por el mero hecho de ser postuladas por la física actual. Por el contrario, si llegase a ser el caso que la física fundamental futura postulase entidades mentales, entonces el fisicalismo sería refutado.

No es difícil ver, sin embargo, que la adición de dicha predicción a la formulación del fisicalismo adolece del mismo problema de nosistematicidad que el requisito de NMF en el marco del enfoque por objeto. De hecho, pese a la introducción de la predicción, sigue siendo cierto en los propios términos de Brown \&o Ladyman que el ectoplasma debería ser considerado físico si la ciencia llegase a incluirlo en su dominio. Y el problema no se atenúa al considerar lo remoto de esa posibilidad: lo que ella muestra es que la predicción es un recurso ad hoc que privilegia sin fundamento aparente unas intuiciones sobre otras con el sólo objeto de

Manuscrito - Rev. Int. Fil. Campinas, v. 40, n. 2, pp. 71-97, abr.jun. 2017. 
salvar el fisicalismo de la trivialidad haciéndolo falsable. Después de todo (al menos para algunos) las entidades mentales son intuitivamente tan ajenas al reino de lo físico como las estructuras constituidas por relaciones sin relata.

Por las razones expuestas sostengo que ambos enfoques fracasan en brindar una caracterización adecuada de lo físico, o al menos una que pueda acomodarse a los propósitos del REO. La salida a ese problema, sostengo, es la articulación de un enfoque mixto.

\subsection{El círculo virtuoso: metafísica naturalizada y ciencia metafísicamente informada}

Hasta el momento hemos explorado críticamente tres escenarios posibles:

A. La filosofía, por sí sola, determina el modo de caracterizar lo físico.

B. La ciencia, por sí sola, determina el modo de caracterizar lo físico.

C. La ciencia determina el modo de caracterizar lo físico, pero la filosofía le impone límites (e.g. el requisito de NMF).

He procurado presentar argumentos clásicos y novedosos para mostrar que ninguna de estas tres opciones es un camino adecuado, al menos en lo concerniente a la caracterización de las estructuras físicas. Hay una obvia cuarta opción a la mano, que no parece haberse encarnado en ninguna propuesta articulada:

D. La filosofía determina el modo de caracterizar lo físico, pero la ciencia le impone límites.

Hay más de un ejemplo histórico que puede ilustrar este punto, pero basta mencionar las intuiciones ligadas al atomismo clásico o las fuerzas de acción a distancia para mostrar como nociones ligadas a formas filosóficas de comprender lo físico pueden ser limitadas o refutadas por desarrollos científicos.

Manuscrito - Rev. Int. Fil. Campinas, v. 40, n. 2, pp. 71-97, abr.jun. 2017. 
Si bien esta posición es pasible de críticas semejantes a las esgrimidas contra $\mathrm{C}$, no me detendré en ese punto ya que, de hecho, el enfoque que propongo en este trabajo es en realidad una combinación entre $\mathrm{C}$ y $\mathrm{D}$ (C/D). Mi propia formulación requiere la identificación de un conjunto de intuiciones filosóficas ligadas a las entidades físicas (IF). El conjunto IF incluye aquellas intuiciones ligadas al carácter no fundamentalmente mental de lo físico, como así también a que lo físico no puede en principio ser de naturaleza abstracta, intensional o espiritual. Sin embargo, no es preciso que seamos capaces de identificar cada uno de los componentes de IF, sino sólo que éstos estén disponibles en el caso de que cierta clase de entidades deba ser sometida a C/D. Mi enfoque sobre IF, además, contempla un aspecto clave, a saber, la composición de IF puede ir variando - como de hecho ha ido variando- a lo largo del tiempo, especialmente a la luz de nuevos desarrollos científicos que acaban por "decantar" al nivel de las intuiciones. En otras palabas, el alto grado de confirmación y legitimación científica de una teoría puede traducirse, en el ámbito de la discusión metafísica, en la necesidad de modificar el contenido de IF para acomodarlo a nuevos desarrollos. Pero, en sentido inverso, la verdad del fisicalismo no queda asegurada desde un principio. La especulación filosófica acerca de la ciencia puede valerse de IF para dictaminar que cierta entidad, incluso siendo postulada por la física vigente, es lo suficientemente divergente de las intuiciones filosóficas aceptadas como para no ser considerada una entidad física. Veamos entonces en qué consiste el enfoque mixto que propongo:

C/D: una entidad es física si y sólo si

(i) es investigada, de modo al menos aproximado, por la física fundamental, $y$

(ii) es (o puede argumentarse convincentemente que resulta) compatible con IF

Esta formulación da cuenta de la idea general detrás de las propuestas de Wilson y Brown \& Ladyman, en el sentido de que la especulación metafísica puede poner límites a lo que la ciencia determine como físico, de modo tal que el contenido de la tesis fisicalista no resulte trivialmente

Manuscrito - Rev. Int. Fil. Campinas, v. 40, n. 2, pp. 71-97, abr.jun. 2017. 
verdadero. Sin embargo, no está afectada por el problema de la no sistematicidad. Si bien se admite que IF puede ir cambiando, y con él C/D, éste siempre brinda condiciones suficientes y necesarias para que una entidad sea considerada física. Se acomoda, a la luz de la historia de la ciencia, a dos fenómenos igualmente retratados por episodios concretos. Primero, al hecho de que nuestras creencias metafísicas, e incluso nuestras intuiciones, pueden cambiar conjuntamente con el desarrollo de la ciencia. Segundo, al hecho de que la especulación filosófica, tal como se la concibe en el marco de la — cada vez más desarrollada - metafísica naturalizada, juega un rol relevante no sólo en la asimilación de los resultados científicos, sino en la producción de conceptos que pueden ser útiles para la ciencia.

Como he tratado de establecer en las secciones precedentes, considero que el REO tiene todavía un importante trabajo por delante a la hora de brindar un relato satisfactorio acerca del carácter físico de las estructuras que postula. Debe quedar claro que mi propuesta no pretende establecerse como una solución a dicho problema, ni siquiera como una alternativa completamente articulada. He intentado, sin embargo, sentar las bases para el desarrollo un marco fisicalista en el que la noción de estructura física pueda ser adecuadamente formulada. Dicho marco no pretende ser funcional sólo a los propósitos de las versiones eliminativistas del REO, sino también constituirse como un terreno fructífero para el desarrollo de versiones no-eliminativistas y otras variantes dentro del cambo de las ontologías estructuralistas para la ciencia.

\section{Conclusión}

La identificación de la estructura del mundo con una pura estructura matemática, esto es, lo que Tegmark denomina HUM, constituye una tesis implausible cuya fundamentación parte de serios errores conceptuales y comete, además, petición de principio. La versión más elaborada del REO hace descasar el carácter físico de las estructuras en dos pilares conceptuales: su naturaleza causal o modal, y una distinción entre la presentación y la representación de las estructuras. He procurado mostrado que los argumentos en este sentido son insatisfactorios.

Manuscrito - Rev. Int. Fil. Campinas, v. 40, n. 2, pp. 71-97, abr.-jun. 2017. 
Por otra parte, el análisis de las posiciones en el debate acerca del fisicalismo muestra que tanto el enfoque por objeto como el enfoque por teoría son insuficientes para fundamentar la noción de estructura física conveniente para el REO y, además, adolecen de problemas difícilmente salvables. El enfoque mixto que propongo hace justicia a la idea de que es la reflexión metafísica la que determina lo que ha de considerarse como físico, al tiempo que salva la intuición de que la metafísica ha de estar limitada y orientada por los resultados de la ciencia. Este nuevo marco fisicalista se presenta como el más adecuado para la formulación de ontologías estructuralistas.

\section{Referencias}

BRADING, K. \& E. LANDRY Scientific structuralism: Presentation and representation. Philosophy of Science 73: 571-581, 2006.

BORGE, B. "¿Qué es el Realismo Estructural Óntico?: una aproximación al debate actual sobre el Realismo Científico". Revista Colombiana de Filosofía de la Ciencia 13 (27): 149-175, 2013.

BROWN, R. \& LADYMAN, J. Physicalism, supervenience and the fundamental level. The Philosophical Quarterly, 59(234), 20-38, 2009.

CAO, T. Y. Can we dissolve physical entities into mathematical structures?. Synthese, 136(1), 57-71, 2003.

Crook, S. \& GilLETT, C. Why physics alone cannot define the 'physical': Materialism, metaphysics, and the formulation of physicalism. Canadian Journal of Philosophy, 31(3), 333-359, 2001.

ESFELD, M. The modal nature of structures in ontic structural realism. International Studies in the Pbilosophy of Science, 23(2), 179-194, 2009.

FRENCH, S. The structure of the world: Metaphysics and representation. Oxford University Press, 2014.

\& Ladyman, J. "Remodelling structural realism: Quantum physics and the metaphysics of structure." Synthese, 136: 31-56, 2003.

Manuscrito - Rev. Int. Fil. Campinas, v. 40, n. 2, pp. 71-97, abr.jun. 2017. 
Hempel, C. 'Reduction: Ontological and Linguistic Facets', in Patrick Suppes, Sidney Morgenbesser and Morgan White (eds.), Philosophy, Science, and Method: Essays In Honor of Ernest Nagel (pp. 179-199),St. Martin’s, 1969.

"Comments on Goodman's Ways of Worldmaking", Synthese XLV, 193-199. 1980.

LADYMAN, J. "What is structural realism?" Studies in History and Philosophy of Science, 29: 409-424, 1998. and Ross, D. (with Spurrett, D. and Collier, J.) Every Thing Must Go: Metaphysics Naturalised, Oxford: Oxford University Press, 2007.

MELnYK, A. "How to Keep the "Physical" in Physicalism", Journal of Philosophy 94, 622-637,01997.

ReICHENBACH, H. The Theory of Relativity and A Priori Knowledge. Translated by Maria Reichenbach. Berkeley: University of California Press, [1920] 1965.

Stoljar, D. 'Physicalism', Stanford On-line Encyclopedia of Philosophy, 2001.

TEgmark, M. The mathematical universe. Foundations of Physics, 38, 101150, 2008.

VAN FraAsSEN, B. C. Representation: The problem for structuralism.Philosophy of Science, 73(5), 536-547, 2006b.

"Structure: Its shadow and substance," The British Journal for the Philosophy of Science, 57: 275-307, 2006a.

WILSON, J. On characterizing the physical. Philosophical Studies,131(1), 6199, 2006.

WOrRALL, J. 'Structural Realism: The Best of Both Worlds?', Dialectica, 43: 99-124, 1989.

Manuscrito - Rev. Int. Fil. Campinas, v. 40, n. 2, pp. 71-97, abr.jun. 2017. 
